\title{
DESIGN OPTIMIZATION AND TRADE-OFFS OF MINIATURIZED WIDEBAND ANTENNA FOR INTERNET OF THINGS APPLICATIONS
}

\author{
Muhammad Aziz ul Haq, Sławomir Koziel \\ Reykjavik University, School of Science and Engineering, 101 Reykjavik, Iceland \\ (\koziel@ru.is,+3545996376,muhammadu16@ru.is)
}

\begin{abstract}
Internet of Things (IoT) will play an important role in modern communication systems. Thousands of devices will talk to each other at the same time. Clearly, smart and efficient hardware will play a vital role in the development of IoT. In this context, the importance of antennas increases due to them being essential parts of communication networks. For IoT applications, a small size with good matching and over a wide frequency range is preferred to ensure reduced size of communication devices. In this paper, we propose a structure and discuss design optimization of a wideband antenna for IoT applications. The antenna consists of a steppedimpedance feed line, a rectangular radiator and a ground plane. The objective is to minimize the antenna footprint by simultaneously adjusting all geometry parameters and to maintain the electrical characteristic of antenna at an acceptable level. The obtained design exhibits dimensions of only $3.7 \mathrm{~mm} \times 11.8 \mathrm{~mm}$ and a footprint of $44 \mathrm{~mm}^{2}$, an omnidirectional radiation pattern, and an excellent pattern stability. The proposed antenna can be easily handled within compact communication devices. The simulation results are validated through measurements of the fabricated antenna prototype.
\end{abstract}

Keywords: compact antennas, Internet of Things, communication devices, numerical optimization, footprintreduction-oriented design.

\section{Introduction}

In the modern era, the internet has revolutionized the way of gathering and processing information. An increasing number of communication devices are connected to the internet owing to their compact size and cost effectiveness. Hence, the concept of Internet of Things (IoT) has been introduced. A widespread use of wireless networks also promotes shift towards modern communication devices rather than traditional ones such as desktop computers, tablets, etc. According to the recent survey, over 50 million such devices will be interconnected with each other via internet by the end of this decade [1-3]. The required hardware structure for IoT devices is different from that for traditional communication networks. Therefore, design and manufacturing processes of these devices should account for their role in the future communication systems and their specific applications. In particular, the advent of IoT calls for new wireless microwave systems which are cost-effective and compact by design, offer high data transmission rates, a low power consumption, so that they could be used in wearable devices [4].

In recent years, there have been research efforts observed towards designing specific antennas for IoT applications with a narrow bandwidth, such as a miniature antenna for IoT [5], a dual band antenna [6], or a compact reconfigurable antenna [7]. However, the selection of a specific antenna for any application may be a real challenge. Some of research efforts are focused on identifying suitable technologies for fifth-generation communication and IoT applications, especially to enable handling all devices by one system [8]. Hence, it is actually 
more important to design antennas which offer a wide frequency range of operation [9]. On the other hand, design of compact antennas for any wireless system is a non-trivial task due to fundamental limitations pertinent to electrically small antennas [10].

In this paper, we propose a structure of a compact monopole antenna for IoT applications. Numerical optimization is performed to minimize the antenna size and to ensure its acceptable matching. The selected frequency range of interest is $5 \mathrm{GHz}$ to $10 \mathrm{GHz}$, important for $5 \mathrm{G}$ technology [11-13]. The selection of the frequency is made due to the advancement in technologies such as $4 \mathrm{G}$ and $5 \mathrm{G}$. The footprint of the optimized antenna is only $44 \mathrm{~mm}^{2}$. Due to its small size and good electrical performance, the proposed antenna can be a good candidate for various IoT applications; in particular, it can be easily mounted on small wireless devices. An alternative version of the antenna with improved matching obtained at the cost of a slight size increase is also considered. The simulation and measurement results confirm good electrical and field properties of both structures.

\section{Antenna structure}

The antenna geometry is shown in Fig. 1. The structure is a modification of a basic rectangular monopole antenna with an additional micro-strip section (parameters $L_{2}, w_{2}$ ) added to serve as an impedance transformer to facilitate wideband matching. The antenna is implemented on Taconic RF-35 substrate $\left(\varepsilon_{r}=3.5, h=0.762 \mathrm{~mm}, \tan \delta=0.0018\right)$. The design variables are $x=\left[\begin{array}{lllllllll}L_{1} & L_{2} & L_{3} & w_{1} & w_{2} & L_{g} & w_{g} d_{L} & d_{w}\end{array}\right]^{\mathrm{T}} ; w_{s}=w_{2}+2 . d_{w} ; w_{0}=2 \mathrm{~mm}$ is fixed to ensure 50-ohm input impedance. The computational model is implemented in CST Microwave Studio [14] ( 200,000 mesh cells, simulation time 2 minutes). The EM model is equipped with an SMA connector in order to enable reliable experimental validation of the design. The antenna is supposed to operate in a frequency range from $5 \mathrm{GHz}$ to $10 \mathrm{GHz}$.

\section{Design optimization}

Our primary goal is to reduce the antenna size while maintaining acceptable matching within a $5 \mathrm{GHz}$ to $10 \mathrm{GHz}$ frequency range. To achieve this, all geometry parameters of the antenna have been adjusted simultaneously through numerical optimization. Let $A(x)=\left(L_{1}+\right.$ $\left.L_{2}+L_{3}+d_{L}\right)$. $w_{s}$ and $S(x)=\max \left\{|\mathrm{S} 11(\mathrm{x})|_{5 \mathrm{GHz}}\right.$ to $\left.10 \mathrm{GHz}\right\}$ be the antenna footprint and EMsimulated maximum in-band reflection level, respectively. In rigorous terms, the objective is to reduce $A(x)$ and ensure $S(x) \leq-10 \mathrm{~dB}$. Thus, the task is to solve ( $c f$. [15]):

$$
\boldsymbol{x}^{*}=\arg \min _{\boldsymbol{x}}\left\{A(\boldsymbol{x})+\beta \cdot c(S(\boldsymbol{x}))^{2}\right\},
$$

where: $\beta$ is a penalty factor (here, $\beta=1000$ ), whereas $c$ is a penalty function defined as $c(S(x))$ $=\max \{\mathrm{S}(\mathrm{x})+10) / 10,0\}$. The penalty term enforces $S\left(x^{*}\right)$ to satisfy the $-10 \mathrm{~dB}$ threshold [15]. In this work, the problem (1) is solved using the trust-region-based [16] gradient search. The finite differentiation is used to estimate the reflection response Jacobian.

For the sake of comparison, the antenna will be also designed for the best possible matching within the frequency range of interest $(5 \mathrm{GHz}$ to $10 \mathrm{GHz})$. In this case, we use a simpler formulation of the following form:

$$
\boldsymbol{x}^{*}=\arg \min _{\boldsymbol{x}}\{S(\boldsymbol{x})\} .
$$

The optimization engine is the same as before (TR-based gradient search with numerical derivatives). 


\section{Results and experimental verification}

In this section we provide the results of antenna optimization. We consider two cases: (i) the miniaturization-oriented optimization, and (ii) the matching-oriented design. The reason for considering the alternative design is to identify the benefits of miniaturization-oriented design in terms of an additional size reduction achieved but also to examine the electrical and field performance differences between the two structures.

The antenna of Fig. 1 has been optimized using the approach described in Section 3. We will refer to the antenna optimized for the minimum size as Antenna I, and to the antenna optimized for the best matching as Antenna II. The detailed antenna dimensions are shown in Tables 1 and 2, respectively. The footprint area of Antenna I is only $44 \mathrm{~mm}^{2}$, whereas the footprint of Antenna II is $69 \mathrm{~mm}^{2}$. Fig. 2 shows photographs of the fabricated antenna prototypes. The simulated and measured reflection responses are shown in Fig. 3, whereas Figs. 4 through 7 show the simulated and measured radiation patterns. Finally, Fig. 8 shows the simulated and measured achieved gain of both antennas. It can be observed that an agreement between the simulation and measurement results is very good. Relatively large discrepancies of E-plane radiation patterns for certain directions are due to the shadowing effect of the 90-degree bend the antenna was mounted on during the measurement process.

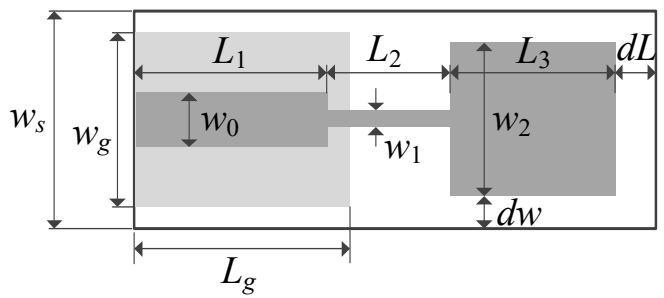

Fig. 1. Geometry of the considered compact antenna. The ground plane is shown using a light grey shade.

a)
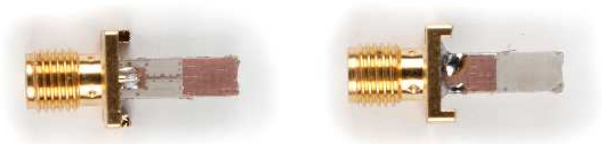

b)
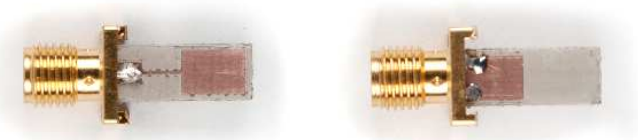

Fig. 2. Photographs of the fabricated antenna prototypes: the antenna optimized for the minimum size (Antenna I) (a); the antenna optimized for the best matching (Antenna II) (b).

The antenna fronts and backs are shown on the left- and right-hand sides, respectively.

Formulating the design problem as described in Section 3 enables to ensure that the optimized design exhibit the smallest size while still satisfying the matching requirements at the prescribed frequency range of $5 \mathrm{GHz}$ to $10 \mathrm{GHz}$. The simulated maximum in-band reflection for Antenna II was about $-14 \mathrm{~dB}$ which leaves a sufficient margin to accommodate such effects as fabrication and assembly tolerances or inaccuracy of the computational model, and still to obtain acceptable matching for the measured prototype. This can indeed be observed in Fig. 3b. On the other hand, Antenna I exhibits a slight violation from the $-10 \mathrm{~dB}$ threshold for frequencies between $9 \mathrm{GHz}$ and $10 \mathrm{GHz}$. 
The H-plane radiation patterns of both antennas are omnidirectional throughout the entire frequency range. For E-plane patterns, as mentioned before, a noticeable difference between the simulation and measurement results can be identified which is a result of the aforementioned shadowing effect. The achieved gain of both antennas is almost linear from $5 \mathrm{GHz}$ to $10 \mathrm{GHz}$. The gain performance is excellent, given a very small footprint size.

a)

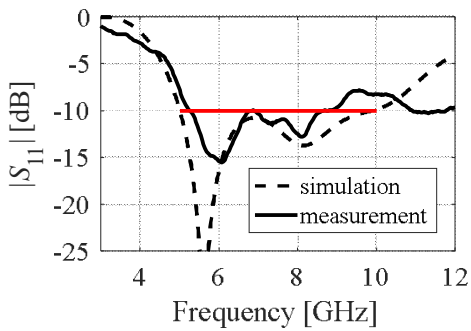

b)

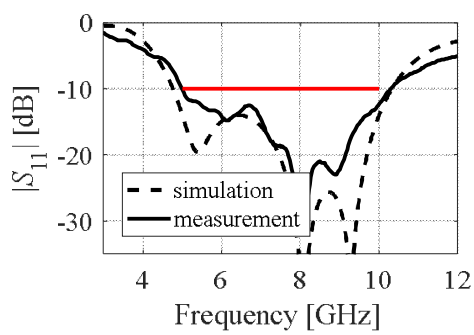

Fig. 3. The simulated and measured reflection characteristics: Antenna I (a); Antenna II (b). The frequency range of operation $(5 \mathrm{GHz}$ to $10 \mathrm{GHz})$ is marked using a horizontal line.
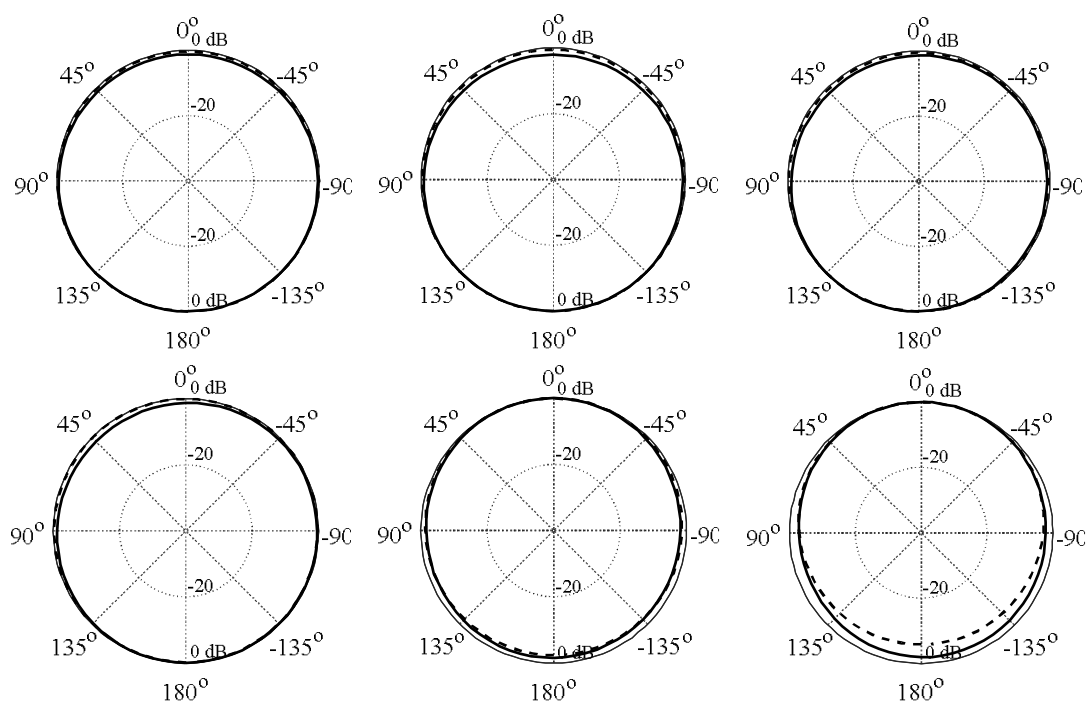

Fig. 4. The simulated and measured H-plane radiation patterns (E field) for the antenna optimized for the minimum size (Antenna I). From left top to bottom right: $5 \mathrm{GHz}, 6 \mathrm{GHz}, 7 \mathrm{GHz}$, $8 \mathrm{GHz}, 9 \mathrm{GHz}$, and $10 \mathrm{GHz}$. 

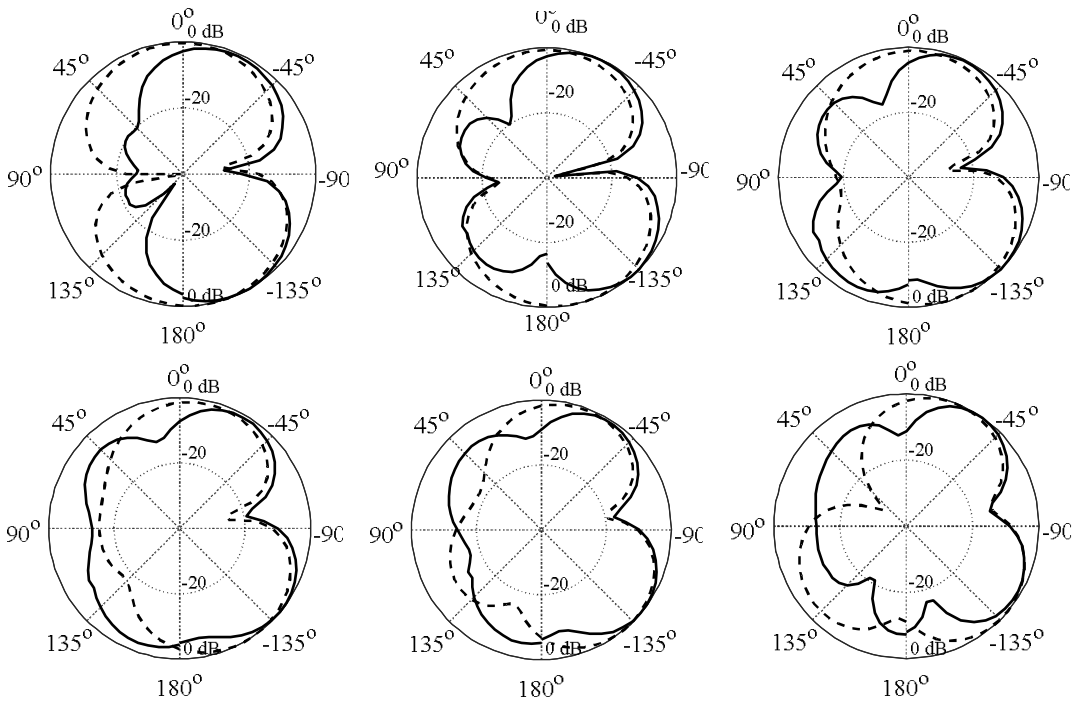

Fig. 5. The simulated and measured E-plane radiation patterns (E field) for the antenna optimized for the minimum size (Antenna I). From left top to bottom right: $5 \mathrm{GHz}, 6 \mathrm{GHz}, 7 \mathrm{GHz}$, $8 \mathrm{GHz}, 9 \mathrm{GHz}$, and $10 \mathrm{GHz}$.
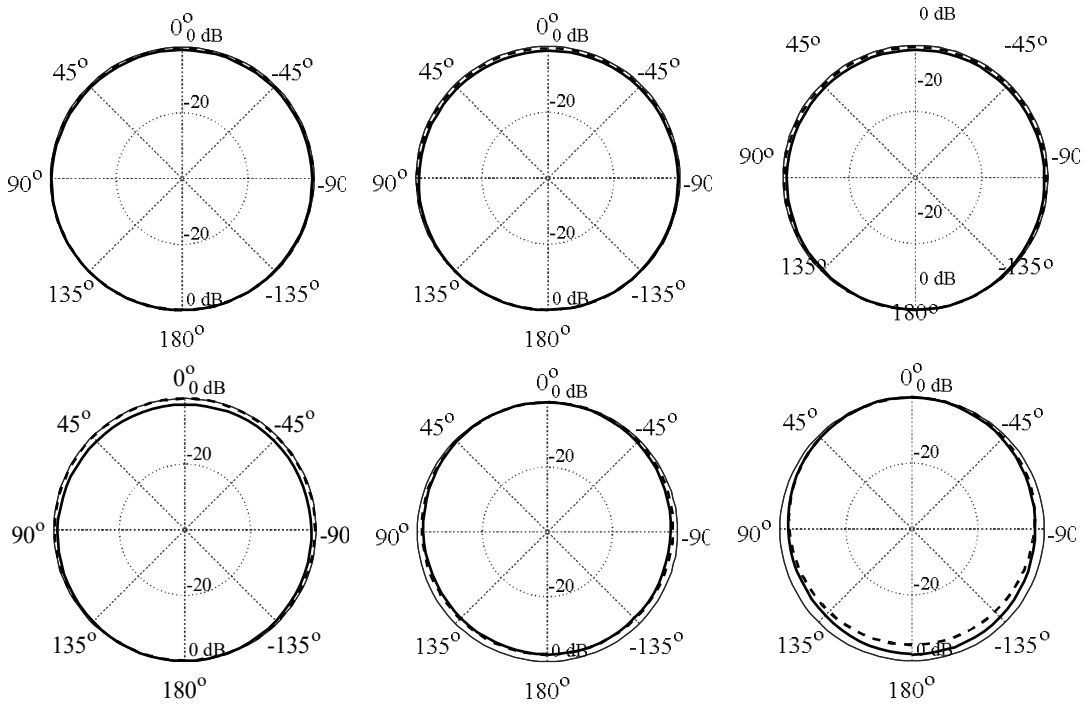

Fig. 6. The simulated and measured $\mathrm{H}$-plane radiation patterns (E field) for the antenna optimized for the best matching (Antenna II). From left top to bottom right: $5 \mathrm{GHz}, 6 \mathrm{GHz}, 7 \mathrm{GHz}$, $8 \mathrm{GHz}, 9 \mathrm{GHz}$, and $10 \mathrm{GHz}$. 

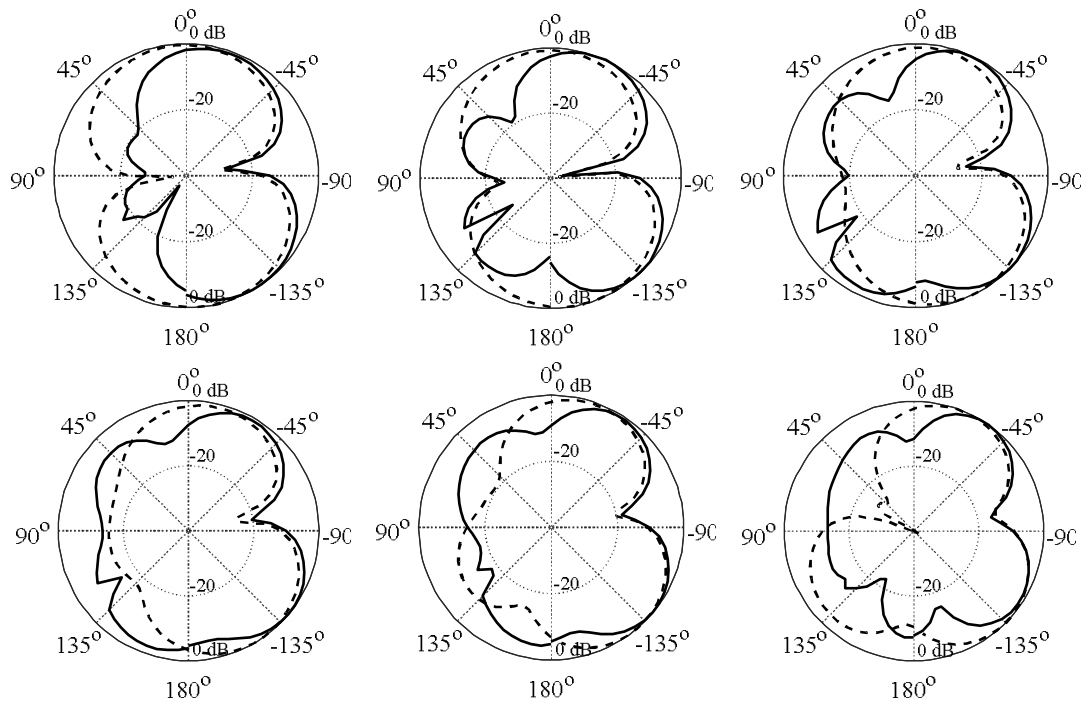

Fig. 7. The simulated and measured E-plane radiation patterns (E field) for the antenna optimized for the best matching (Antenna II). From left top to bottom right: $5 \mathrm{GHz}, 6 \mathrm{GHz}, 7 \mathrm{GHz}$, $8 \mathrm{GHz}, 9 \mathrm{GHz}$, and $10 \mathrm{GHz}$.

Table 1. Optimized Dimensions (Antenna I).

\begin{tabular}{|c|c|c|c|c|c|c|c|c|c|}
\hline Variable & $L_{1}$ & $L_{2}$ & $L_{3}$ & $w_{1}$ & $w_{2}$ & $L_{g}$ & $w_{g}$ & $d L$ & $d w$ \\
\hline Value $[\mathrm{mm}]$ & 2.70 & 3.74 & 5.36 & 0.20 & 3.74 & 5.45 & 3.73 & 0.00 & 0.00 \\
\hline
\end{tabular}

Table 2. Optimized Dimensions (Antenna II).

\begin{tabular}{|c|c|c|c|c|c|c|c|c|c|}
\hline Variable & $L_{1}$ & $L_{2}$ & $L_{3}$ & $W_{1}$ & $w_{2}$ & $L_{g}$ & $w_{g}$ & $d L$ & $d w$ \\
\hline Value $[\mathrm{mm}]$ & 2.43 & 3.91 & 6.05 & 0.28 & 4.24 & 5.63 & 4.17 & 0.63 & 0.54 \\
\hline
\end{tabular}

a)

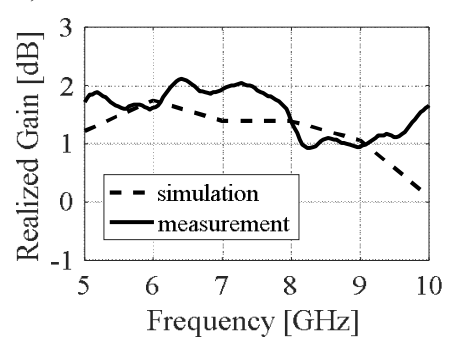

b)

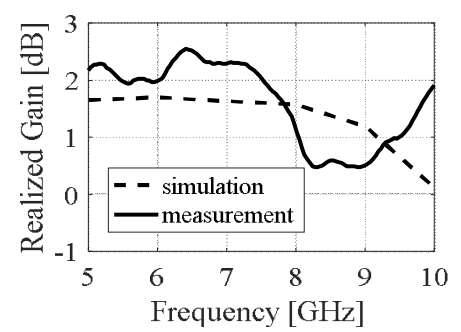

Fig. 8. The simulated and measured achieved gain: the antenna optimized for the minimum size (a); the antenna optimized for the best matching (b).

Figure 7 shows the simulated total efficiencies of the antennas. Clearly, the efficiency of Antenna II is higher than that of Antenna I. Nevertheless, the average in-band total efficiencies for both antennas are over 90\% (92\% for Antenna I and 96.5\% for Antenna II).

The optimized antennas have been also compared in respect to the pattern stability, using the pattern stability factor (PSF) [17] that represents the relationship between radiation in a reference direction and radiation in all other directions over a specified bandwidth; here, 
the operational bandwidth of the antenna. As indicated in [17], for a good performance of a UWB system, $P S F$ of 0.95 or larger is desired. $P S F$ is defined as:

$$
P S F=\int_{\Omega} C(\vec{R}) d s / \int_{\Omega} d s,
$$

where: $\Omega$ is a range of operating directions (here, following [17], the H-plane is considered, so that the integral in (3) is reduced to a linear one); and $C(\vec{R})$ is a frequency-domain correlation pattern defined as [17]:

with

$$
C(\vec{R})=\int_{\Omega} F^{2}(\vec{R}, \vec{r}) d s / \int_{\Omega} d s
$$

$$
F^{2}(\vec{R}, \vec{r})=\left|\int_{B W} E(\vec{r}, f) E^{*}(\vec{R}, f) d f\right| /\left[\int_{B W}\left|E(\vec{r}, f)^{2}\right| d f \int_{B W}\left|E^{*}(\vec{R}, f)\right|^{2} d f\right],
$$

where: $E(\vec{r}, f)$ is a far-field electric field in the direction $\vec{r} ; \vec{R}$ is a reference direction; and * denotes a complex conjugate.

The PSF values for Antenna I and Antenna II are 0.956 and 0.962, respectively. This means that miniaturization of the antenna structure (specifically, Antenna I over Antenna II) has no negative effect on the pattern stability, similarly as concluded in [18].

Finally, in order to verify the robustness of the design, three copies of each antenna have been fabricated. The measured reflection responses have been shown in Fig. 8. It can be observed that the characteristics closely resemble each other, indicating that the designs are robust in respect to manufacturing and assembly tolerances.

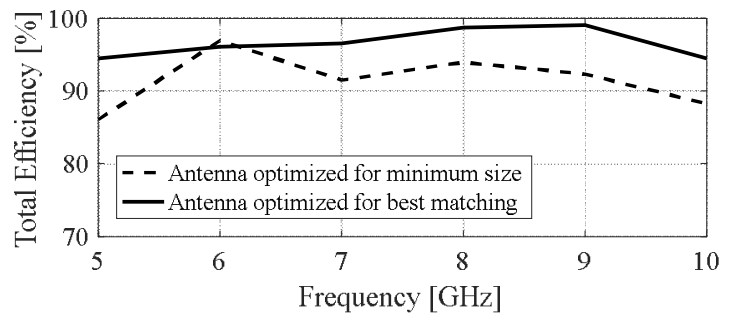

Fig. 9. Comparison of total efficiencies of Antenna I and Antenna II.

a)

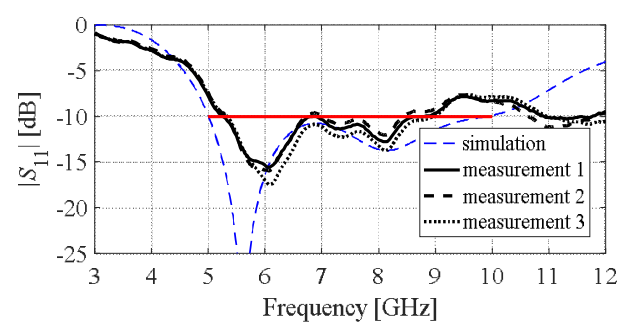

b)

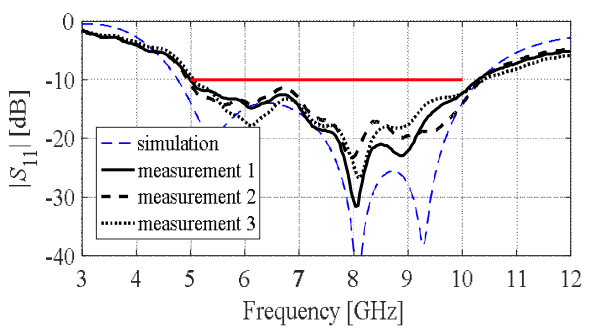

Fig. 10. The simulated and measured reflection characteristics: the antenna optimized for the minimum size (a); the antenna optimized for the best matching (b). The results of measurements 1 through 3 correspond to three copies of the fabricated antennas.

The frequency range of operation $(5 \mathrm{GHz}$ to $10 \mathrm{GHz})$ is marked using a horizontal line. 


\section{Conclusion}

In the paper, the structure and design optimization of a miniaturized wideband antenna for Internet of Things applications is presented. Simultaneous adjustment of all geometry parameters of the antenna enables to achieve a very small footprint of only $44 \mathrm{~mm}^{2}$ and a good matching within the prescribed frequency range of $5 \mathrm{GHz}$ to $10 \mathrm{GHz}$. At the cost of certain increase of its size (to $69 \mathrm{~mm}^{2}$ ), the antenna matching can be improved by around $4 \mathrm{~dB}$. The discussed structures exhibit a good electrical and field performance, including high efficiency, omnidirectional radiation pattern, high pattern stability, and almost linear gain.

\section{Acknowledgment}

The authors thank Computer Simulation Technology AG, Darmstad Germany for making CST Microwave Studio available. This work is partly supported by the Icelandic Centre for Research (RANNIS) Grant 163299051.

\section{References}

[1] Grau, A. (2016). How to Build a Safer Internet of Things: Todays IoT is full of security flaws. We must do better. IEEE Spectrum, http://spectrum.ieee.org/telecom/security/how-tobuild-a-safer-internet-of-things.

[2] Kamilaris, A., Pitsillides, A. (2016). Mobile Phone Computing and the Internet of Things: A Survey. IEEE Internet of Things Journal, 885-898.

[3] Jin, J., Gubbi, J., Marusic, S., Palaniswami, M. (2014). An Information framework for creating smart city through internet of things. IEEE Internet of Things Journal, 112-121.

[4] Moscato, S., Silvestri, L., Delmonte, N., Pasian, M., Bozzi, M., Perregrini, L. (2016). SIW components for the Internet of Things: Novel topologies, materials, and manufacturing techniques. IEEE Topical Conference on Wireless Sensors and Sensor Networks (WiSNet), 78-80.

[5] Lizzi, L., Ferrero, F., Monin, P., Danchesi, C., Boudaud, S. (2016). Design of miniature antennas for IoT applications. IEEE International Conference on Communications and Electronics, 234-237.

[6] Katoch, S., Jotwani, H., Pani, S., Rajawat, A. (2015). A compact dual band antenna for IOT applications. International Conference on Green Computing and Internet of Things, 1594-1597.

[7] Mansour, A.M., Mokhtar, B., Gomah, K., Marghany, K., Abdelmonsef, A. Rizk, M.R.M., Shehata, N. (2016). Compact reconfigurable multi-size pixel antenna for cognitive radio networks and IoT environments. Loughborough Antennas \& Propagation Conference, 1-5.

[8] Zanella, A., Bui, N., Castellani, A., Vangelista, L., Zorzi, M. (2014). Internet of things for smart cities. IEEE Internet of Things Journal, 22-32.

[9] Khan, M.A., ul Haq, M.A., ur Rehman, S. (2016). A practical miniature antenna design for future internet of things enabled smart devices. Int. Conf. Signal Processing and Communication Systems.

[10] Bekasiewicz, A., Koziel, S. (2016). Compact UWB monopole antenna for internet of things applications. Electr. Lett., 52, 492-494.

[11] Livingston, V., Pearson, C., Svensson, A., Arefi, R. (2015). 4G Americas' vision for the americas: 5G spectrum recommendations. http:// http://www.4gamericas.org/files /1914/ 412 2/0345/5G_Spectrum_ Recommendations_Webinar_9.2.2015.pdf.

[12] Lemey, S., Caytan, O., Vande Ginste, D., Demeester, P., Rogier, H., Bozzi, M. (2016). SIW cavity-backed slot (multi-)antenna systems for the next generation IoT applications. IEEE Topical Conference on Wireless Sensors and Sensor Networks, 75-77.

[13] Palattella, M.R., Dohler, M., Grieco, A., Rizzo, G., Torsner, J., Engel, T., Ladid, L. (2016). Internet of things in the 5G Era: enablers, architecture, and business models. IEEE J. Selected Areas in Communications, 510-527. 
Metrol. Meas. Syst., Vol. 24 (2017), No. 3, pp. 463-471.

[14] CST Microwave Studio, ver. 2015. CST AG, Bad Nauheimer Str. 19, D-64289 Darmstadt, Germany, 2015.

[15] Bekasiewicz, A., Koziel, S. (2015). Structure and computationally-efficient simulation-driven design of compact UWB monopole antenna. IEEE Ant. Wireless Prop. Lett., 14, 1282-1285.

[16] Conn, A.R., Gould, N.I.M., Toint, P.L. (2000). Trust Region Methods. MPS-SIAM Series on Optimization.

[17] Dissanayake, T., Esselle, K.P. (2006). Correlation-based pattern stability analysis and a figure of merit for UWB antennas. IEEE Trans. Ant. Prop., 54(11), 3184-3191.

[18] Liu, J., Esselle, K.P., Hay, S.G., Zhong, S. (2014). Effects of printed UWB antenna miniaturization on pulse fidelity and pattern stability. IEEE Trans. Ant. Prop., 62(8), 3903-3910. 